\title{
La transmición del psicoanálisis en el camino de la participacion ${ }^{1}$ \\ María Alejandra Vázquez, Gabriela Ruíz Irrazábal, María Noel Mauttoni, Mariana Mantiñán y Ximena Méndez ${ }^{2}$
}

\section{Un poco de historia.}

Podríamos decir, si es que existe un comienzo que a finales de la década del '40 un grupo de profesionales iniciaron la búsqueda. Camino largo y no poco escabroso, sobre la base del deseo de analizarse y hacerse analistas. Desde allí, la búsqueda de la transmisión del psicoanálisis, los llevó (y aún nos lleva) por distintos caminos que nuestros antecesores transitaron buscando la transmisión del psicoanálisis. El grupo de interesados en transitar dicha experiencia crecía, es así que en la década del '50 Willy y Madeleine Baranger se radican en el Uruguay a fin de posibilitar la formación teórica y análisis didáctico de aquellos que los convocaban. Durante este proceso al que Mercedes Freire de Garbarino llamó la "prehistoria de la APU” el apoyo brindado por nuestros colegas argentinos fue fundamental, para que en adelante pudiésemos andar nuestros propios caminos.

En la década del '60 fuimos reconocidos por la IPA. Pero fue a partir del XII Congreso Latinoamericano que se realizara en Uruguay en 1966, donde mediante un arduo trabajo conjunto, al decir de Mercedes F. de Garbarino “...sentíamos que a partir de él adquiríamos nuestra mayoría de edad.” (1988, p.10)

Aquí ya podemos ver como se dibuja la historia de APU desde el deseo de transitar por la experiencia analítica, la transmisión del psicoanálisis, la formación teórica y práctica supervisada, y la participación esa que desde los inicios de la Asociación constituye un pilar fundamental y fundante.

\footnotetext{
1 Trabajo presentado en XI Congreso Argentino de Psicoanálisis, "Escenarios del Psicoanálisis", mayo 2018 y en I Simposio de la Sociedad Brasilera de Psicoanálisis de San Pablo, "O mesmo, O outro: Psicanálise em movimento", agosto 2018. Eixo "Psicanaálise e suas clínicas", Mesa "O mesmo e o outro na formação em Psicanálise” com Luis Carlos Menezes e Eduardo São Thiago Martins

${ }^{2}$ Analistas en formación del Instituto de Postgrado en Psicoanálisis de APU.
} 
Entre el 1972 y 1974 se realizan cambios entre los que se destaca la desconcentración del poder del "didacta". Hasta este momento el analista didacta centralizaba todas las funciones: análisis, supervisión, docencia y era quien autorizaba o no a sus analizandos el ingreso al Instituto. A partir de aquí el análisis personal quedaba separado de toda incidencia institucional en lo que respecta a la formación. Se crean además los llamados grupos de funciones ${ }^{3}$, que buscaba una democratización mayor; teniendo por objetivo fundamental el sostén institucional del ejercicio de la función. Así mismo la comisión de Enseñanza pasó a estar integrada también por candidatos. En palabras de Javier García

Fue democratizador, siguiendo un modelo de nuestra universidad, incluyendo a los candidatos en la comisión de enseñanza y formando los grupos de funciones didácticas que descentralizaban el poder de la persona del didacta para distribuirlo a nivel grupal. Esos fueron cambios importantes y se constituyeron en un rasgo particular del Instituto uruguayo. (2015, p.157)

Desde los años '90 la comisión que estudiaba el plan de formación trabajó en la construcción de cambios que apuntaban a que los candidatos participen en el diseño de su recorrido por el Instituto. Así en los seminarios se integran las diferentes generaciones, con los distintos recorridos y experiencias. Tanto la libre elección como la decisión del número de seminarios a cursar (siempre dentro del plan de estudios), implica entonces al candidato de una manera activa. También se brinda libertad a los docentes a fin de presentar creativamente y sobre la base de sus intereses propuestas de seminarios, grupos de estudio, etc.

Surge desde distintos sectores de APU el interés de que el Instituto sea reconocido como Instituto Universitario de post grado en Psicoanálisis. Se crea una comisión que representa en el Ministerio de Educación y Cultura a APU, logrando el reconocimiento

\footnotetext{
${ }^{3}$ Grupos de reflexión, de estudio e investigación con funcionamiento horizontal que cuentan con la participación de representantes de los candidatos.
} 
del Instituto de Psicoanálisis de APU como Instituto Universitario de Post- Grado en Psicoanálisis. ${ }^{4}$

Otro aspecto que nos parece importante destacar en tanto característico del modelo uruguayo, son algunas de las condiciones y requisitos en los que se da el ingreso al Instituto. En cuanto a los requisitos para la postulación se encuentran: hallarse en un análisis personal de alta frecuencia (mínimo 3 sesiones semanales) con un analista habilitante del Instituto, poseer título universitario de Psicólogo o Médico Psiquiatra y presentar un currículum que a juicio del interesado de cuenta de sí mismo y de su recorrido hasta el momento de la postulación. El aspirante deberá presentar su solicitud por carta a la Comisión de Enseñanza, quien la derivará a la Comisión de Admisión del Instituto para dar curso a la misma. Cada postulante será entrevistado por dos analistas integrantes de dicha comisión, en forma individual y consecutiva. El objetivo de las entrevistas es que el aspirante pueda dar cuenta de su deseo, sus razones, su proyecto de formación como analista entramado con su historia y su proceso de análisis (información confidencial). Una vez que dicha comisión se expide, será la Comisión de Enseñanza quien comunicará al interesado si su solicitud de ingreso al Instituto ha sido o no aceptada.

\section{Puertas abiertas}

Un aspecto que se ha venido trabajando intensamente con muy buena receptividad, es el diálogo con la comunidad, "APU de puertas abiertas a la comunidad", transitando y haciendo transitar por distintos escenarios al psicoanálisis. Espacios culturales, sociales, científicos y artísticos. Ejemplos de estas actividades son "El Bar de Freud”, Ciclo de Cine y Teatro con posterior debate e intercambio, Encuentros con el Autor, Exposiciones de Pintura, Ciclos de entrevistas en Televisión Nacional de Uruguay donde se dialoga con la audiencia. También se realizan Actividades Científicas y se

${ }^{4}$ El 17 de julio de 2003 se constituye como Instituto Universitario por el reconocimiento del Ministerio de Cultura. El 31 de mayo de 2007 se autoriza el registro del título de Máster en Psicoanálisis por el Ministerio de Salud Pública. 
dictan cursos abiertos tanto presenciales como on-line. Es evidente la necesidad de tener a mano los elementos que surgen de la cultura, cine, teatro, literatura, música, pintura son escenarios que desde Freud y hasta hoy hemos recorrido, utilizándolos como puentes, como andamios en la transmisión del psicoanálisis.

En este sentido es fundamental el trabajo de APU en la Universidad. Es a destacar que el Instituto de posgrado de APU (IUPP) cuenta desde hace tres años con un Seminario del Área libre de Freud (área V) que se realiza en el Hospital Maciel en el marco de la Clínica Psiquiátrica de la Facultad de Medicina, bajo la forma de Ateneo Psicoanalítico interdisciplinario, coordinado por docentes del IUPP; en el que participan psiquiatras, residentes, candidatos, psicólogos y estudiantes de Psicología, asistentes sociales, y estudiantes de dicha carrera, enumeración que no pretende ser exhaustiva, pero que da idea del interés que despierta el trabajo desde la interdisciplina y la receptividad que ha tenido en el ámbito universitario la participación de APU.

Desde los inicios (atravesados y atravesando los distintos escenarios culturales, la prehistoria, la historia y la actualidad de APU) la búsqueda ha sido por el sendero de la transmisión del psicoanálisis y sus pilares no negociables: el inconsciente, la sexualidad y la transferencia. Por un lado la intensa experiencia en transferencia del análisis personal, por otro la formación teórica y la práctica supervisada, y por último, la articulación de las tres atravesada por la participación.

\section{Nos interrogamos}

Como candidatos, ¿Incide nuestra participación en el devenir institucional? y de ser así, ¿Cómo? ¿Qué efectos tendría la convivencia que implica la participación, el trabajo conjunto, si es que efectivamente lo hace? (pensemos en las transferencias cruzadas, los riesgos de lo endogámico, por ejemplo) ¿Qué se va dando entre el cómo llegamos y en qué advenimos una vez que hacemos el pasaje por la Institución? 
Tomaremos para el inicio del presente trabajo que hoy queremos compartir con ustedes, uno de tantos aspectos, el de ser recibidos en la Institución por candidatos de otras generaciones, qué huella deja y la importancia de pensar sobre ello. El porqué de la dimensión que cobra para algunos ese momento casi inaugural que por su carácter de estar inscripto en el tiempo, irá dando lugar a otros momentos. Sin duda no se podría dar cuenta de ello sin respetar la singularidad de cada recorrido. A pesar de lo difícil de poner en palabras tal experiencia lo intentaremos, con las limitaciones inevitables.

Consideramos que el tiempo que antecede a la entrada al Instituto (análisis, deseo de ser analista, decisión de presentarse, entrevistas de ingreso y finalmente la admisión coronada con la carta que cada uno de nosotros recibe: "De nuestra consideración, Comunicamos a Ud. que de acuerdo a lo informado por la Comisión de Admisión, su solicitud de ingreso al Instituto de Psicoanálisis ha sido aceptada" Atentamente y firma del Director del Instituto) da lugar a otra entrada que es la de ir entrando a la Institución, y ello está marcado entre otras cosas (por lo menos para nosotras) por el recibimiento de los compañeros que ya vienen haciendo los seminarios y que nos vamos enterando prontamente que están agrupados en la Organización de Candidatos de la Asociación Psicoanalítica del Uruguay (OCAPU); recibimiento que sólo más adelante se resignificará y dará lugar, entre otras cosas, a esta reflexión.

Pensamos, ¿esta forma de darnos la bienvenida tendrá el efecto en la generación que entra de calmar (disipar) fantasías persecutorias, multiplicidad de afectos, temores que traemos desde hace un tiempo, más o menos extenso según la peripecia de cada uno, pero siempre movilizante? Clima previo al ingreso que quedaría definido por múltiples cuestionamientos: ¿estaremos a la altura?, ¿nos transformaremos en alguien a la medida de la Institución? Entramos, sí; nos admiten, sí ¿a qué? o ¿para qué?, ¿saldremos formateados? ¿En el sentido de organizados según un formato para ser operativos? Temores que por un instante casi de júbilo quedan subsumidos por el alivio de un recibimiento que alimenta fantasías de unificación, que calma las vivencias de exclusión, desagregación, etc. 
Nuestros anfitriones nos van contando de sus experiencias en el tránsito por la formación, el recorrido por los diversos seminarios, la preparación de notas, trabajos, incertidumbres, y nos ofrecen su apoyo tanto en cuestiones administrativas como institucionales y académicas (jugando con la palabra anfitrión la usamos en el sentido de recibir y compartir lo que es suyo, sin olvidar el deslizamiento a la acepción de anfitrión como el que trata de apagar cualquier posible tensión que pueda surgir entre los invitados); comenzando así a resignificar y establecer lazos que hacen a la fratría. Se subraya en todos lo fundamental de la existencia y vigor de lo que damos en llamar entre nosotros la cuarta pata de la formación; entendiendo por dicha expresión la trayectoria institucional que los integrantes de OCAPU van teniendo en la medida que participan del devenir institucional.

Desde allí, y en medio de la calidez y entusiasmo al recibirnos en lo que se irá transformando en nuestra casa, nuestro lugar de pertenencia; recibimiento que destacamos y que nos motiva a brindarlo a las generaciones que vengan; pero calidez también que nos podría quemar si negamos lo que pulsa en todo grupo humano, en donde la pertenencia trae de suyo el temor al desconocido, al que viene de afuera, los narcisismos puestos en juego, roces, rivalidades fraternas, vivencias de exclusión, aspectos que de no ser tenidos en cuenta nos podrían hacer caer en el terreno de la ilusión del deseo satisfecho, sensaciones de completud, reminiscencias infantiles reanimadas toda vez que talla la angustia de lo que no conocemos, ¿vivencias de desamparo?

Vamos percibiendo el compromiso, el involucramiento con la Institución. Una Institución que nos recibe (y de la que esperamos ir siendo parte) un ser parte que hay que ir construyendo, en el recorrido. La participación del analista en formación en los distintos espacios del Instituto tiene un gran valor formativo permitiendo lugares de inserción, de acción y de reflexión. Somos llamados a la participación, implicación activa, que nos da paso a ir ocupando lugares, pero que tenemos que conquistar y poblar desde nuestra singularidad; en los tiempos de cada uno, al ritmo de cada uno, en un diseño a medida pero también conjugando un compromiso con lo institucional que nos 
solicita; solicitación a la que podemos acceder o no, pero en caso de responder al llamado, se va generando una pertenencia conjugada en gerundio, por lo tanto inacabada pero sostenida en proceso. Pasajes diversos, cada uno con peripecias distintas que hacen a las diferencias, las que tenemos que saber apreciar y darles asidero para propiciar el enriquecimiento de diversas posturas, la emergencia de los posibles debates e intercambios que van tapizando el recorrido por la Institución. Trabajar sobre la prioridad de, como candidatos, ser motores de un pensamiento reflexivo, de interrogarnos sobre la Institución, la que es y la que queremos; que las discusiones den lugar a acciones promotoras de cambio, y estar advertidos de no quedar entrampados en la quietud propia de toda institución que tiende a la inercia; Institución-Institucionalidad que por necesaria (en tanto tercero que ayuda a desatar intrincados atravesamientos transferenciales) no apague la vocación por cuestionarnos, de reflexionar sobre nuestra formación. Que el entusiasmo de participar y la asunción de desafíos, la tolerancia a no saber y la frustración propia del pensamiento freudiano, sean nuestra brújula.

Dice Susana García en las palabras de apertura al primer congreso de analistas en formación de agosto de 2010 “(...) creo que esto [el esfuerzo de participar] tiene que ver con el análisis y nuestra formación como analistas: nuestra tarea implica siempre un compromiso personal grande y en el mejor de los casos una gratificación diferida". (2010, p.9)

Pronto nos encontramos en la lista de correo que agrupa a los candidatos, los que ingresan, los que están, todos, y allí se encuentra uno de los lugares donde se percibe la actividad, intereses, cuestionamientos, organización y participación de jornadas, encuentros, respuestas a otras organizaciones de candidatos de otros países. Instancias que hacen a convocar reuniones regulares y en esos encuentros darnos cuenta, poco a poco, como la asistencia va dando lugar a la participación y así a través de este ámbito, se va entrando a lo Institucional. Muchas veces nos encontramos con la sensación de que la Institución nos va requiriendo a un ritmo por momentos difícil de seguir, sentimos lo bueno de ser convocados pero al mismo tiempo se vive la frustración de no poder con todo -vivencias de ser demandados y no poder dar respuesta a todo lo que nos presenta la Institución, generando ansiedades en muchos de nosotros por no cumplir con 
los requisitos de APU. Lo que nos mueve también a pensar sobre nuestros propios límites, aceptar la castración ${ }^{5}$. Por desplazamiento, surge el mismo sentimiento con la agrupación de candidatos que nos alberga, y así APU/OCAPU quedarían en el lugar de aquello que detenta un ideal y por ende nos sentimos constreñidos a una adhesión para no perder los privilegios que en una suerte de pacto implícito nos da la Institución (amparo, inclusión, identidad). Creemos que estos itinerarios al compartirlos en diversos ámbitos, este es uno de ellos, nos ayudan a estar advertidos de los riesgos de no reflexionar sobre nuestro camino en la formación y en la trasmisión del psicoanálisis, dado que estos ideales circulan también de una forma inconsciente (referimos a los ideales puestos en la Institución, que podrían sustituir nuestro narcisismo perdido de la infancia donde nosotros mismos éramos nuestro propio ideal).

Desde aquel lugar inicial de ser recibidos, espectadores - expectantes de ver cómo trabajan nuestros compañeros que ya traen un recorrido en la Institución, nos vamos apropiando de un lugar que se nos da y que tendríamos la oportunidad de ir ocupando. En ese ir haciendo de un transitar, una pertenencia, vamos asumiendo responsabilidades, el compromiso con la Institución y sus avatares, Institución que nos 'admite' pero en ese mismo movimiento tenemos la chance desde la participación de incidir (para aquellos que nos sentimos llamados a ello, que no necesariamente tiene que ser todo el colectivo).

En las palabras de apertura al primer congreso de candidatos que recién mencionábamos, Susana García ${ }^{6}$ hablando de la necesaria idealización de la Institución a la que aspiramos y de la función a desarrollar como futuros analistas, menciona también la importancia de que esa idealización se atenúe para poder advenir como analistas, dice textualmente:

[...] sabemos que es muy tentador el poder de la transferencia [decimos nosotros transferencia institucional, transferencias múltiples, con

\footnotetext{
${ }^{5}$ Por eso la idea del nuevo plan del IUPP de propiciar que cada candidato diseñe su propio recorrido en la formación.

${ }^{6}$ En su condición de Directora científica de APU y en esa oportunidad como presidenta del IV congreso de APU.
} 
docentes, supervisores, analistas] sabemos que es muy fácil sostener el camino del narcisismo y seguir considerando nuestro conocimiento y nuestra experiencia, no como algo a dar, no como don a entregar, sino como algo detrás de lo que nos abroquelamos y considerar a los demás como 'faltos de experiencia, necesitan más análisis, demasiado jóvenes...' y es allí, donde ustedes [refiere a nosotros, los candidatos] tienen que dar batalla (ídem. p.10-11)

Allí queremos detenernos. Batallar en el sentido del compromiso que asumimos con la Institución (APU) desde OCAPU, entendiendo por ello el trabajo sostenido de confrontar con la postura que ubicaría a APU en el lugar de una Institución ideal generadora de un modelo de analista ideal y una formación reglamentada que lo garantice, y en esa confrontación ir desmontando lo que hemos ubicado en ese lugar idealizado, para poder emerger como sujetos críticos, participantes activos en nuestra formación (sujetos de nuestra formación); abogar por una trasmisión del psicoanálisis, trasmisión que nos remitiría a pasar de un lugar a otro, ser atravesados por, en el entretejido de múltiples transferencias, con la Institución, con nuestros docentes, supervisores, transferencias que hacen a la formación, transferencias cruzadas que hacen obstáculo y que también tendrían que caer para finalmente advenir como analistas portadores de lo analítico en donde la incompletud y relatividad del saber le es inmanente así como el rechazo a toda certidumbre que trae la amenaza de lo dogmático. El cuestionamiento permanente propio del Psicoanálisis tanto desde su teoría como desde su práctica nos guían en nuestra formación y las palabras de Freud a Fliess "A veces algo dentro de mí, me impulsa hacia una síntesis, pero yo me resisto" (Freud, S. 1887-1904, p.447) nos ayudan a estar atentos.

Cada uno de los candidatos tenemos nuestros tiempos, ritmos y modos de ir transitando los requerimientos institucionales, la organización en la que nos agrupamos (OCAPU), que integramos pero que también nos precede, que oficia muchas veces de red de contención, de ámbito donde hacer decir nuestras incertidumbres, malestares, no está libre de convertirse en un lugar que tiende a homogeneizar, donde plantear disidencias y ser portavoz de disconformidades del funcionamiento grupal no siempre son de buen recibo; esto nos lleva a trabajar, y a estar precavidos de no enlentecernos hasta la quietud, de quedar parapetados en un discurso de apertura a lo nuevo, a lo diverso que 
podría disfrazar fuertes resistencias al cambio, a la complejidad. OCAPU nos presta un lugar a referirnos en el camino (peripecia) de la formación, en una formación que nos mueve e interpela sin pausa y creemos así tiene que ser, la revisión de nuestro funcionamiento como organización, el pensarnos en nuestro quehacer, creemos favorece la fortaleza de OCAPU.

Este irnos impregnando de lo institucional a través de las diferentes comisiones que integramos con candidatos que nos representan, comisiones que nos sirven de puente con la Asociación Psicoanalítica y sus representantes, va haciendo en nosotros una suerte de transformación desde nuevas identificaciones, que va dando lugar a lo que Javier García llama 'hacerse analista', advenimiento que creemos tiene un grado de parentesco con la participación, el ser/sintiéndose parte, la implicancia, el esfuerzo y compromiso, que para nosotros hace a la trasmisión del Psicoanálisis.

Pensamos este 'hacerse analistas' no desde lo que se nos dicta, como adiestramiento o instrucción, sino desde pasajes, tránsitos, itinerarios, que van dejando huella, marcas nuevas que, nos atrevemos a decir, va sucediendo en un ir siendo atravesados por diversas transferencias, lo inconsciente y sus expresiones, lo que nos va impulsando; un ir siendo/ir haciéndose, que tiene por condición para mantenerse vivo el que no se establezca de una vez y para siempre y que nos permite hacer de nuestra pasión por el psicoanálisis nuestro derrotero.

Desde esta impronta de advenir analistas, recordamos que los candidatos también somos analistas en formación. Cómo somos nominados por nosotros mismos y por los otros, hace a quienes somos. ¿Será momento de comenzar a llamarnos analistas en formación desde un lugar formal? Situación que se da de hecho en varios ámbitos no formales. ¿Será momento de institucionalizar lo que venimos sintiendo y proponiendo? ¿De alguna forma, no estamos siendo parte ya de la transformación de OCAPU en OAF de APU, Organización de Analistas en Formación de APU? Creemos que es parte de nuestra participación en la transmisión del psicoanálisis poder reconocer que lo que 
adviene como analistas es mucho más que nosotros mismos. Esperamos que en un horizonte no muy lejano podamos sustituir en los trabajos que presentamos la nominación candidato por analista en formación, cambio a conquistar desde el trabajo y discusión en la organización y en el diálogo con la asociación.

Para Freud [...] "el progreso del conocimiento no tolera rigidez alguna, tampoco en las definiciones. Como lo enseña palmariamente el ejemplo de la física, también los conceptos básicos fijados en definiciones experimentan un constante cambio de contenido" (1915, p.113)

Nos dice Javier García en su ensayo "Transmisión Institucionalizada del Psicoanálisis...":

[...] Parece importante, entonces, en medio de tantas fuerzas que hacen difícil la producción de un sujeto, hacer todo lo posible para sostener ese delicado lugar de gestación de cada analista en la singularidad de cada experiencia". "[...] [la] singularidad de la formación de cada aspirante y candidato es una pequeña delicada joya a cuidar, a preservar, cultivándola para hacer producir a esta diversidad. (García, J. 2014, p.142)

Y junto con él ubicamos este aspecto artesanal y distinguible como uno de los rasgos esenciales de la formación. "El desafío es incluir las diferencias” (ídem.)

Consideramos para finalizar, que al trípode que sustenta la formación desde el modelo uruguayo, conformado por: análisis personal, formación teórica y la formación en la práctica supervisada, añadimos la participación en nuestra organización de candidatos; a través de ella y de nuestros representantes en las distintas comisiones- y nuestra implicación en el quehacer institucional, desde dentro, desde el trabajo del día a día, en la marcha, marcha que supone apertura a lo nuevo desde lo que nos antecede (la historia, lo que hace raíz, nos funda y nos lanza a desafíos) lugar de referencia que nos espeja identidad y pertenencia a nuestra Institución que es ésta y no otra. 
Y en este sentido de lo nuevo y lo que nos viene desde los que nos preceden, nos gustaría hacer nuestras las palabras de Marcelo Viñar en relación a la trasmisión en psicoanálisis:

Hemos importado de la antropología (y/o historia de las ideas) la importancia de la transmisión entre generaciones, y de lo único que estamos seguros es de su existencia y eficacia simbólica en el progreso de la ciencia y la cultura, aún cuando ignoremos buena parte de los itinerarios, andariveles y laberintos por donde se produce esta transmisión, entre continuidades y rupturas. (Pérez, L. 2011, p.139)

\section{Referencias}

Freire de Garbarino, M. (1988) Breve historia de la Asociación Psicoanalítica del Uruguay. ISSN 1688-7247. Revista uruguaya de psicoanálisis (En línea) (68)

Freud, S. Cartas a Wilhelm Fliess (1887-1904) Sigmund Freud. Editorial Amorrortu. Buenos Aires. 1994

Freud, S. (1915) Obras completas. Editorial Amorrortu. Buenos Aires. 1992, V.XIV

García, J. (2015) Conversando con Javier García. Grafo No. 14.

García, J. (2014) La transmisión institucionalizada del psicoanálisis en los comienzos del siglo XXI. Revista Uruguaya de Psicoanálisis No. 118

García, S. (2010) Primer Congreso de Analistas en formación. Palabras inaugurales de apertura. Grafo No. 10.

Viñar, M. (2010) Comentario sobre el texto de Ricardo Bernardi. Nota sobre la obra de G. Koolhaas. A propósito de la recepción de las ideas de Lacan en el Río de la Plata. En Revista Uruguaya de Psicoanálisis No. 111 